\title{
Average value of the cosmic ray injection exponent at Galactic sources
}

\author{
Anatoly Lagutin ${ }^{\mathrm{a}}$ and Nikolay Volkov ${ }^{\mathrm{b}}$ \\ Altai State University, Radiophysics and Theoretical Physics Department, 656049, 61 Lenin ave, Barnaul, Russia
}

\begin{abstract}
The energy spectrum of high energy cosmic rays emitted by sources change during propagation in the Galaxy. Using the spectrum observed at the Earth and two different transport models, based on anomalous and normal diffusion equations, we have retrieved an average value of the cosmic ray injection exponent at Galactic sources taking into account the inhomogeneity of the interstellar medium. We have shown that the average value of the injection index $p$, obtained in the framework of both transport models, equals to $p \sim(2.8-3.0)$.
\end{abstract}

\section{Introduction}

The spectrum of cosmic rays (CRs) measured at the Earth is different from their source spectrum. A key to understanding this difference is the determination of how cosmic ray (CR) particles propagate through the turbulent interstellar medium (ISM).

Assuming that the medium is quasi-homogeneous, the propagation process is described by GinzburgSyrovatsky's normal diffusion equation $[1,2]$

$$
\frac{\partial N(\mathbf{r}, t, E)}{\partial t}=D(E) \Delta N(\mathbf{r}, t, E)+S(\mathbf{r}, t, E)
$$

where $N(\mathbf{r}, t, E)$ is the density of particles with energy $E$ at the location $\mathbf{r}$ and time $t, D(E)=D_{0} E^{\delta}$ is the diffusion coefficient assumed to be spatially constant and $S(\mathbf{r}, t, E)$ is the source term.

The steady state solution of (1), frequently used for interpretation of the $\mathrm{CR}$ phenomena, has the form

$$
N(\mathbf{r}, E) \sim E^{-p-\delta},
$$

where $p$ is the injection spectrum exponent at the Galactic sources. The same result may be obtained using the entirely uniform Leaky-Box model.

The steady state energy spectrum (2) leads to a very simple rule to retrieve the exponent $p$ from the spectrum $N(E) \propto E^{-\eta}$ observed at the Earth: $p=\eta-\delta$. Since $\eta \approx$ 2.7 , for $\delta \sim 0.3$ we find that the slope of the CR injection spectrum at the source is $\sim 2.4$.

However, recent results show that homogeneous diffusion models, based on (1), failed to reproduce observations (see, for example, [3]). Multiscale structures in the Galaxy, found during the last few decades, may be taken as a support to this conclusion.

Theory and observations show that the ISM is inhomogeneous (fractal-like) on the hundreds of parsecs

\footnotetext{
a e-mail: lagutin@theory.asu.ru

b e-mail: volkov@theory. asu.ru
}

scales [4-6]. Stars formation regions also demonstrate fractal features with spatial scales up to about a kpc [7-9].

The particles emitted by Galactic sources en route to the Solar system pass through regions of the Galaxy that have different properties. In such a inhomogeneous ISM, the normal diffusion model is certainly not kept valid.

The main goal of this paper is to retrieve the cosmic ray injection exponent at the Galactic sources from the spectrum observed at Earth taking into account the inhomogeneity of the turbulent interstellar medium. Two different models, based on anomalous and normal diffusion equations, are used to relate the spectrum injected by the source and that measured at Earth.

In the following section we give an overview of the anomalous diffusion model, discuss our injection exponent retrieval technique and the results obtained in this model. In Sect. 3 we present a new approach to describe cosmic ray propagation in the non-homogeneous ISM, based on the normal diffusion Eq. (1). Our findings and conclusions are given in Sect. 4.

\section{Retrieval of the injection exponent using the anomalous diffusion model}

The non-homogeneous character of matter distribution and associated magnetic field leads to the need to incorporate these ISM features into the cosmic ray diffusion model. A possible way to generalize the normal diffusion model is to replace the assumption about statistical homogeneity of inhomogeneities distribution by their fractal distribution. An important consequence of this generalization is the power-law distribution of free paths, $r$, in such a medium $p(\mathbf{r}, E) \propto A(E, \alpha) r^{-\alpha-1}, r \rightarrow \infty, 0<\alpha<2-$ so-called Lévy flights. Since an intermittent magnetic field of the fractal-like ISM leads to nonzero probability of a long stay of particles in inhomogeneities, the presence of the Lévy trap can not be excluded. In the general case, the probability density function $q(t, E)$ of time, $t$, during which a particle is trapped in the inhomogeneity

(C) The Authors, published by EDP Sciences. This is an Open Access article distributed under the terms of the Creative Commons Attribution License 4.0 (http://creativecommons.org/licenses/by/4.0/). 
(Lévy trap), also has a power-law behavior: $q(t, E) \propto$ $B(E, \beta) t^{-\beta-1}, t \rightarrow \infty, \beta<1$.

A generalization of the homogeneous normal diffusion model to the case of inhomogeneous (fractal-like) ISM, has been made for the first time in our papers $[10,11]$. Later, it was shown [12-17] that an anomalous cosmic ray diffusion model, developed by the authors, allows to describe the main features of nuclei, electron and positron spectra observed in the Solar system. Particularly, in the anomalous diffusion model the key feature of the all particle energy spectrum — the knee at $3 \cdot 10^{15} \mathrm{eV}$ appears naturally without additional assumptions.

The equation for the density of particles with energy, $E$, at the location, $\mathbf{r}$, and time, $t$, generated in a fractal-like medium by Galactic sources with a distribution density $S(\mathbf{r}, t, E)$ can be written as $[12,14]$

$$
\begin{aligned}
\frac{\partial N(\mathbf{r}, t, E)}{\partial t}= & -D(E, \alpha, \beta) \mathrm{D}_{0+}^{1-\beta}(-\Delta)^{\alpha / 2} N(\mathbf{r}, t, E) \\
& +S(\mathbf{r}, t, E) .
\end{aligned}
$$

Here $\mathrm{D}_{0+}^{\mu}$ denotes the Riemann-Liouville fractional derivative [18] and $(-\Delta)^{\alpha / 2}$ is the fractional Laplacian ('Riesz operator') [18]. The anomalous diffusion coefficient $D(E, \alpha, \beta) \sim A(E, \alpha) / B(E, \beta)=D_{0}(\alpha, \beta) E^{\delta}$.

The solution of Eq. (3) for a point instantaneous source with a power-law injection spectrum $S(\mathbf{r}, t, E)=$ $S_{0} E^{-p} \delta(\mathbf{r}) \delta(t)$ has the form $[12,14]$

$$
\begin{aligned}
N(\mathbf{r}, t, E)= & S_{0} E^{-p}\left(D(E, \alpha, \beta) t^{\beta}\right)^{-3 / \alpha} \\
& \times \Psi_{3}^{(\alpha, \beta)}\left(|\mathbf{r}|\left(D(E, \alpha, \beta) t^{\beta}\right)^{-1 / \alpha}\right),
\end{aligned}
$$

where $\Psi_{3}^{(\alpha, \beta)}(\rho)$ is the density of the fractional stable distribution $[19,20]$

$$
\Psi_{3}^{(\alpha, \beta)}(\rho)=\int_{0}^{\infty} g_{3}^{(\alpha)}\left(r \tau^{\beta}\right) q_{1}^{(\beta, 1)}(\tau) \tau^{3 \beta / \alpha} d \tau .
$$

Figure 1 demonstrates the behaviour of the function $\Psi_{3}^{(\alpha, \beta)}(\rho)$ for different parameters $\alpha$ and $\beta$.

The asymptotic behaviour of the scaling function $\Psi_{3}^{(\alpha, \beta)}(\rho)$ for $\alpha<2, \beta<1$,

$$
\begin{aligned}
& \Psi_{3}^{(\alpha, \beta)}(\rho) \propto \rho^{-(3-\alpha)}, \quad \rho \rightarrow 0, \\
& \Psi_{3}^{(\alpha, \beta)}(\rho) \propto \rho^{-3-\alpha}, \quad \rho \rightarrow \infty,
\end{aligned}
$$

is shown on Fig. 2.

Note that in the energy range below the knee the cosmic ray density is

$$
N(\mathbf{r}, t, E) \sim \frac{E^{-p+\delta}}{r^{3+\alpha}} .
$$

Taking into account that $\rho \equiv|\mathbf{r}|\left(D(E, \alpha, \beta) t^{\beta}\right)^{-1 / \alpha}$, we find

$$
N \sim E^{-\eta}=E^{-p+\delta}, \quad E \ll E_{k}
$$

and

$$
N \sim E^{-\eta}=E^{-p-\delta}, \quad E \gg E_{k},
$$

where $E_{k}$ is the knee energy. Since $\Delta \eta=\left.\eta\right|_{>E_{k}}-\left.\eta\right|_{<E_{k}}$ is known from experimental data to equal $\Delta \eta \sim 0.6$, the last

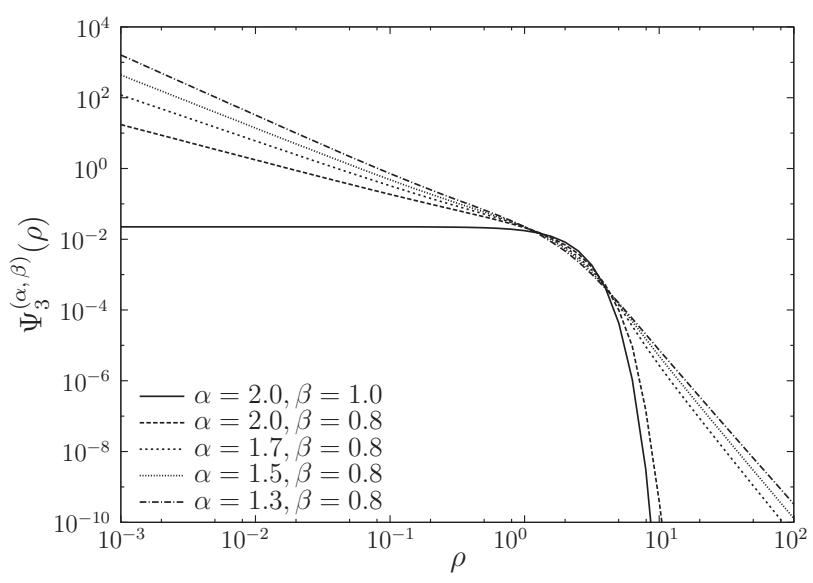

Figure 1. Probability density of fractional stable distribution $\Psi_{3}^{(\alpha, \beta)}(\rho)$ for different parameters $\alpha$ and $\beta$.
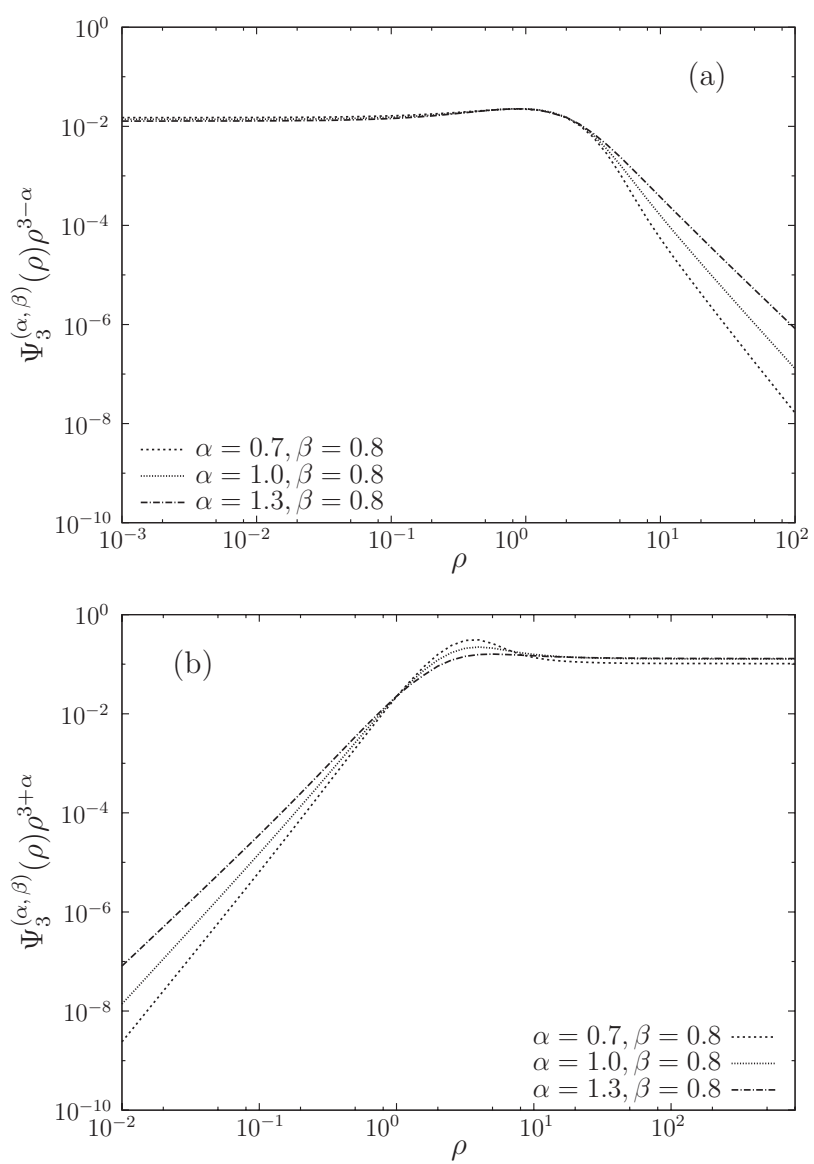

Figure 2. Asymptotic behavior of the function $\Psi_{3}^{(\alpha, \beta)}(\rho)$ for $\rho \rightarrow 0$ (a) and for $\rho \rightarrow \infty$ (b).

equations permit to, self-consistently, retrieve both spectral exponents $p$ and $\delta$ :

$$
\delta=\Delta \eta / 2 \sim 0.3
$$

$$
p=\left.\eta\right|_{<E_{k}}+\delta=\left.\eta\right|_{>E_{k}}-\delta \approx 2.8 \div 2.9 \text {. }
$$

At $E=E_{k}$ the spectral index $\eta\left(E_{k}\right)$ is equal to the injection exponent $p$ at the Galactic source. 


\section{Normal diffusion of cosmic rays in a non-homogeneous ISM}

The medium properties of an inhomogeneous ISM vary in space and time. The particles emitted by Galactic sources en route to the Solar system pass through regions of the Galaxy that have different diffusion coefficients.

Here we suppose that the particles with probability density $\varphi\left(D_{0}\right)$ propagate in the ISM with a diffusion coefficient $D_{0}$. Since for a homogeneous ISM, with the constant diffusion coefficient $D_{0}$, the solution of a normal diffusion Eq. (1) for a point instantaneous source with a power-law injection spectrum has the form

$$
N(\mathbf{r}, t, E)=\frac{S_{0} E^{-p}}{\left(4 \pi D_{0} E^{\delta} t\right)^{3 / 2}} \exp \left(-\frac{r^{2}}{4 D_{0} E^{\delta} t}\right),
$$

we can find the density of particles visiting this part of the Galaxy,

$$
d N_{D}(\mathbf{r}, t, E)=\frac{S_{0} E^{-p} \varphi\left(D_{0}\right) d D_{0}}{\left(4 \pi D_{0} E^{\delta} t\right)^{3 / 2}} \exp \left(-\frac{r^{2}}{4 D_{0} E^{\delta} t}\right),
$$

and then the observed density $N(\mathbf{r}, t, E)$

$$
\begin{aligned}
N(\mathbf{r}, t, E)= & \int d N_{D}(\mathbf{r}, t, E)=\frac{S_{0} E^{-p-3 \delta / 2}}{(4 \pi t)^{3 / 2}} \\
& \times \int_{0}^{\infty} \frac{1}{D_{0}^{3 / 2}} \exp \left(-\frac{r^{2}}{4 D_{0} E^{\delta} t}\right) \varphi\left(D_{0}\right) d D_{0} .
\end{aligned}
$$

In order to have insight into possible types of diffusivity distribution we follow the approach presented in [21]. If $r$ is the random jump length of a particle and $\tau$ is the time required to make this jump, the diffusion coefficient is given by equation

$$
D_{0}=\frac{r^{2}}{2 \tau}
$$

Since $r / \tau$ is the speed $v$, we have

$$
D_{0}=\frac{v^{2} \tau}{2}
$$

Assuming that the speed of particle motion $v$ along its trajectory is the same for all particles, for $\varphi\left(D_{0}\right)$ we find

$$
\varphi\left(D_{0}\right)=\psi(\tau)\left|\frac{d \tau\left(D_{0}\right)}{d D_{0}}\right|=\frac{2}{v^{2}} \psi(\tau) .
$$

It is seen, for example, that the assumption that the time, $\tau$, of a single step is distributed normally leads to a normal distribution for diffusivity. In the case of $\psi(\tau) \propto$ $\tau^{-\beta-1}, \beta<1$ the power-law distribution for $\varphi(D)$ may be obtaned.

Similar distributions may be obtained if we assume that all particles during the random walk have the same value of $r$. In this case $\tau\left(D_{0}\right)=r^{2} /\left(2 D_{0}\right)$ and

$$
\varphi\left(D_{0}\right)=\psi(\tau)\left|\frac{d \tau\left(D_{0}\right)}{d D_{0}}\right|=\frac{r^{2}}{2 D_{0}^{2}} \psi(\tau) .
$$

Using, for example, a normal distribution for $\tau$

$$
\psi(\tau)=\frac{1}{\sqrt{\pi} \Delta \tau} \exp \left[-\left(\frac{\left(\tau-\tau_{0}\right)}{\Delta \tau}\right)^{2}\right],
$$

where $\tau_{0}$ and $\Delta \tau$ are parameters $\left(\Delta \tau \ll \tau_{0}\right)$, for large $D_{0}$ from (7) we find

$$
\varphi\left(D_{0}\right) \sim D_{0}^{-2}
$$

The probability distribution function $\varphi\left(D_{0}\right)$ has a powerlaw form.

Here we consider the power-law distribution $\varphi\left(D_{0}\right) \sim$ $D_{0}^{-\gamma}$ for $D_{0} \rightarrow \infty$, where $\gamma$ is a positive parameter. Since $\varphi\left(D_{0}\right)$ has the meaning of a probability density, that is $\int_{0}^{\infty} \varphi\left(D_{0}\right) d D_{0}=1$, we must assume that $\gamma>1$.

In order to calculate the integral in Eq. (6), we need to make some additional assumptions about the properties of $\varphi\left(D_{0}\right)$ at small and also intermediate values of $D_{0}$. We adopt the following functional form

$$
\varphi\left(D_{0}\right)=A D_{0}^{-\gamma} \exp \left(-\frac{\mathfrak{D}}{D_{0}}\right)
$$

where $A=\frac{\mathfrak{D}^{\gamma-1}}{\Gamma(\gamma-1)}$ is the scaling factor, $\mathfrak{D}$ is the characteristic diffusivity for the Galactic medium, $\gamma>1$ is an auxiliary parameter, $D_{0}>0$. We additionally define $\varphi(0)=0$. find

Having substituted equation (8) into equation (6) we

$$
\begin{aligned}
N(\mathbf{r}, t, E)= & \frac{S_{0} A E^{-p-3 \delta / 2}}{(4 \pi t)^{3 / 2}} \\
& \times \int_{0}^{\infty} \frac{d D_{0}}{D_{0}^{\gamma+3 / 2}} \exp \left[-\left(\mathfrak{D}+\frac{r^{2}}{4 E^{\delta} t}\right) \frac{1}{D_{0}}\right] .
\end{aligned}
$$

Using new variables $p=\mathfrak{D}+\frac{r^{2}}{4 E^{\delta} t}$ and $D_{0}=\frac{p}{z}$, the integral in (9) is transformed as follows:

$$
\begin{aligned}
& \int_{0}^{\infty} D_{0}^{-\gamma-3 / 2} \exp \left(-\frac{p}{D_{0}}\right) d D_{0} \\
& \quad=-p \int_{\infty}^{0} \frac{p^{-\gamma-3 / 2}}{z^{-\gamma-3 / 2}} \exp (-z) \frac{d z}{z^{2}} \\
& \quad=p^{-\gamma-1 / 2} \int_{0}^{\infty} z^{\gamma-1 / 2} \mathrm{e}^{-z} d z=p^{-\gamma-1 / 2} \Gamma\left(\gamma+\frac{1}{2}\right) .
\end{aligned}
$$

As a result, we obtain

$$
\begin{gathered}
N(\mathbf{r}, t, E)=\frac{S_{0} A E^{-p-3 \delta / 2}}{(4 \pi t)^{3 / 2}}\left(\mathfrak{D}+\frac{r^{2}}{4 E^{\delta} t}\right)^{-\gamma-1 / 2} \\
\times \Gamma\left(\gamma+\frac{1}{2}\right)
\end{gathered}
$$


At any given moment of time, $t$, and for sufficiently large $r$, so that $r^{2} \gg 4 \mathfrak{D} E^{\delta} t$, the density of particles, $N(\mathbf{r}, t, E)$, has the form

$$
N(\mathbf{r}, t, E)=\frac{S_{0} \mathfrak{D}^{\gamma-1}}{\pi^{3 / 2}(4 t)^{1-\gamma}} \frac{\Gamma\left(\gamma+\frac{1}{2}\right)}{\Gamma(\gamma-1)} \frac{E^{-p-\delta+\delta \gamma}}{r^{2 \gamma+1}}
$$

or

$$
N(\mathbf{r}, t, E) \sim \frac{E^{-p-\delta+\delta \gamma}}{r^{2 \gamma+1}} .
$$

Thus, it can be seen, that the function $\varphi\left(D_{0}\right)$ given by equation (8) leads to an anomalous spatial distribution of the particle density. Instead of the Gaussian distribution of particle density (5), which is predicted by the homogeneous normal diffusion models, the large-distance asymptotical behavior, we have obtained, is described by a power law $\sim r^{2 \gamma+1}$.

In the discussed model (10) the energy spectrum observed at Earth is $N(E) \propto E^{-\eta}=E^{-p-\delta+\delta \gamma}$. From the slope $\eta=p+\delta(1-\gamma)$ we can find the injection spectrum exponent, $p$, at the Galactic source of the cosmic rays: $p=\eta-\delta(1-\gamma)$. It differs from the steady state energy spectrum exponent $p=\eta-\delta$. Since $\gamma>1$, we have $p>\eta$.

It should be noted that a similar anomalous spatial distribution of the particle density may be found in the case of $\varphi\left(D_{0}\right) \sim D_{0}^{\theta} \exp \left(-\frac{D_{0}}{\mathfrak{D}}\right)$, where $\mathfrak{D}$ is the characteristic diffusivity for the Galactic medium and $0<\theta<1$. This distribution describes a model with exponential decrease at $D_{0} \rightarrow \infty$. For this scenario we have found

$$
N(\mathbf{r}, t, E) \sim r^{\theta-1} \exp \left(-\frac{r}{\sqrt{\mathfrak{D} E^{\delta} t}}\right) E^{-p-\delta(\theta+1) / 2} .
$$

\section{Conclusions}

We have performed the retrieval of the cosmic ray injection exponent at the Galactic sources from the spectrum observed at Earth taking into account the inhomogeneity of the turbulent interstellar medium. Two different transport models, based on anomalous and normal diffusion equations, have been used to relate the spectrum injected by the source with that measured at Earth.

Using the normal diffusion model, we have shown that the variation of the ISM properties leads to an anomalous spatial distribution of the particle density. Its asymptotic behavior (11) for $1.5<\gamma<2$ practically coincides with our result (4) obtained in the framework of the anomalous diffusion model for $1<\alpha<2$. In other words, the cosmic rays observed at Earth collectively appear to display non-diffusive (superdiffusive) characteristics although an individual particle has moved in a diffusive manner.

We have shown that the average value of the injection index $p$, obtained in the framework of both transport models, equals $p \sim(2.8 \div 3.0)$.

We note that a similar steep spectrum of accelerated particles has been observed from supernova remnants
W44 [22] and IC 443 [23] with the Fermi Large Area Telescope. The value $p=3$ has been found in [24] for RX J1713.7-3946.

The authors acknowledge support from The Russian Foundation for Basic Research grant No. 16-02-01103.

\section{References}

[1] V.L. Ginzburg, S. I. Syrovatskii, Origin of cosmic rays (Pergamon Press, 1964)

[2] V.S. Berezinskii, S. V. Bulanov, V. A. Dogiel et al., Astrophysics of Cosmic Rays (Amsterdam, NorthHolland, 1990)

[3] G. Jóhannesson, R. Ruiz de Austri, A. C. Vincent et al., ApJ 824 (2016)

[4] B.G. Elmegreen, J. Scalo, Annu. Rev. Astron. Astrophys. 42, 211 (2004)

[5] E.A. Bergin, M. Tafalla, Annu. Rev. Astron. Astrophys. 45, 339 (2007)

[6] N. Sánchez, E. J. Alfaro, ApJ. Suppl. Ser. 178, 1 (2008)

[7] Y.N. Efremov, A. D. Chernin, Physics - Uspekhi, 46(1), 1 (2003)

[8] R. de la Fuente Marcos, C. de la Fuente Marcos, ApJ 700, 436 (2009)

[9] N. Sánchez, N. Añez, E. J. Alfaro, M. C. Odekon, ApJ 720, 541 (2010)

[10] A.A. Lagutin, Y.A. Nikulin, V.V. Uchaikin, Preprint ASU (2000/4)

[11] A.A. Lagutin, Y.A. Nikulin, V.V. Uchaikin, Nucl. Phys. B (Proc. Suppl.) 97, 267 (2001)

[12] A.A. Lagutin, V.V. Uchaikin, Nucl. Instrum. Meth. B201, 212 (2003)

[13] A.D. Erlykin, A.A. Lagutin, A.W. Wolfendale, Astropart. Phys. 19, 351 (2003)

[14] A.A. Lagutin, A.G.Tyumentsev, Bulletin of Altai State University 5, 4 (2004 in Russian)

[15] A.A. Lagutin, A.V. Yushkov, A.G. Tyumentsev, IJMP A 20, 6834 (2005)

[16] A.A. Lagutin, N.V. Volkov, A.S. Kuzmin, A.G. Tyumentsev, Bull. of RAS: Physics 73(5), 581 (2009)

[17] N.V. Volkov, A.A. Lagutin, A.G. Tyumentsev, J. Phys. Conf. Ser. 632, 012027 (2015)

[18] S.G. Samco, A.A. Kilbas, O.I. Marichev, Fractional integrals and derivatives - Theory and Applications (Gordon and Breach, New York 1993)

[19] V.V. Uchaikin, V.M. Zolotarev, Chance and Stability (VSP, Netherlands, Utrecht, 1999)

[20] V.M. Zolotarev, V.V. Uchaikin, V.V. Saenko, JETP 88(4), 780 (1999)

[21] S.V. Petrovskii, A.Y. Morozov, Am. Nat. 173, 278 (2009)

[22] A.A. Abdo, M. Ackermann, M. Ajello et al., Science 327, 1103 (2010)

[23] A.A. Abdo, M. Ackermann, M. Ajello et al., ApJ 712, 459 (2010)

[24] T. Tanaka, Y. Uchiyama, F. Aharonian et al., ApJ 685, 988 (2008) 